\title{
Editorial
}

\section{The risk of coronary occlusion is not proportional to the prior severity of coronary stenoses}

As clinicians, we treat symptomatic patients with coronary artery disease manifesting as either angina, anginal-like discomfort, or exertional dyspnoea. In some of these patients, symptoms progress rapidly either to rest pain and unstable angina or myocardial infarction, or to sudden death from malignant arrhythmias. However, not all patients who present with these acute coronary syndromes necessarily have a history of symptoms. In fact, in some studies the majority of such patients was asymptomatic before the acute syndrome. The acute progression of a "silent" atherosclerotic plaque to complete or nearly complete occlusion is a common substrate for an acute presentation of coronary artery disease in patients with and without prior symptoms. This editorial discusses the fact that progression to the acute presentation of coronary disease is not proportionately related to the prior severity of the coronary stenosis.

\section{Acute coronary syndromes as secondary or primary presentations of coronary artery disease \\ SYMPTOMATIC PATIENTS \\ Symptomatic patients with known coronary disease often die from myocardial infarction or die suddenly because of the acute progression of a lesion to coronary occlusion. Unfortunately, the mere fact that symptoms were present tells us little about the subsequent acute event as symptoms are not necessarily related to the plaque that progressed and was responsible for the acute event. ${ }^{1-3}$ Even the presence of ischaemia on non-invasive testing may not help in risk stratification for future acute coronary events. On follow up of a stable group of patients with a positive exer- cise stress test or evidence of ischaemia on ambulatory monitoring, Mulcahay et al reported that ischaemia could not predict the future occurrence of an acute coronary syndrome. ${ }^{4}$}

\section{ASYMPTOMATIC PATIENTS}

If we presume that progression to an acute syndrome is not related to the prior severity of a coronary stenosis, it is reasonable that in many patients an acute presentation might be the first manifestation of coronary disease. In fact, an acute syndrome particularly in men is the first symptomatic clinical manifestation of coronary disease in 50-60\% of cases. ${ }^{5}$ In most of these, the first manifestation was acute myocardial infarction but sudden death may occur in $10-25 \%$ of cases without prior symptoms.

\section{Pathophysiology of acute coronary syndromes}

At least one mechanism for this inability to predict future acute events in patients with coronary artery disease is that such events are unrelated to prior severity of a coronary stenosis. Acute coronary events, which include the diagnoses of unstable angina, acute myocardial infarction, and some cases of sudden death, are related to a sudden disruption, fissure or erosion of an atherosclerotic plaque with the formation of an intracoronary thrombus that partially occludes (a majority of patients with unstable angina) or totally occludes (most patients with myocardial infarction or sudden death) the coronary artery. These so called vulnerable plaques, prone to thrombosis, usually contain a large lipid core and a thin fibrous cap with an abundance of inflammatory cells. ${ }^{67}$ These inflammatory cells are generally macrophages capable of degrading the fibrous cap and expressing tissue factor, which contributes to the formation of thrombus. Several angiographic studies in which more than one angiogram was performed on patients who subsequently developed acute syndromes have shown that a majority of these syndromes appears to develop from lesions that on the first angiogram caused less than significant stenoses. ${ }^{23}$

Retrospective studies in patients with acute myocardial infarction or unstable angina indicate that $78-97 \%$ of lesions were less than $75 \%$ obstructed on the initial angiogram. We found less than $50 \%$ narrowing in $48 \%$ of subsequent infarctions on serial angiography, ${ }^{2}$ while in a study by Little et al the incidence was $66 \%{ }^{3}$ A recent angiographic study found progression of coronary disease on a second angiogram in $89 \%$ of patients with an acute syndrome and $74 \%$ of the lesions caused less than $50 \%$ stenosis on the first angiogram. ${ }^{4}$ Although these studies have selected out a population of patients in whom serial angiography was performed, the results differ significantly from patients who had been restudied and found to have a new total occlusion but without an intervening infarction. In this latter group, a severe lesion with greater than $70 \%$ narrowing is found in the majority of patients on the first angiogram.

In addition to these retrospective studies, angiographic studies performed after successful thrombolysis often show moderate coronary stenoses of the infarct related artery. Brown et al found less than $50 \%$ or $60 \%$ stenosis in $66 \%$ of lesions after myocardial infarction, similar to the incidence reported by other investigators. ${ }^{8}$ In a recent postmortem study in which Mann and Davies performed angiography postmortem to assess the stenoses of so called lipid rich vulnerable plaques, there was no correlation between percentage diameter stenosis and the presence of these lesions. ${ }^{9}$ These pathological data support the concept that stenosis severity is not an important determinant of future acute coronary events.

Even though angiographic studies, because of the nature of angiography, will underestimate the degree of atherosclerosis in comparison to either pathological studies or the use of newer techniques like intravascular ultrasound, the concept that certain high grade stenotic lesions remain stable over long periods of time is a valid concept, while lesions progressing to acute coronary syndromes are often not severely obstructed before disruption. It is these less than severely stenotic lesions that lead to infarction when an occlusive thrombus forms as collateral vessels cannot be acutely recruited to prevent or limit the extent of myocardial necrosis. These minor or silent plaques are also more frequently seen in patients with multivessel disease and may partially explain why long term mortality is higher with multivessel rather than single vessel disease. ${ }^{10}$ The more plaques present, the higher is the chance that one or more might be sites for future acute coronary events. 
While angiography is limited in its ability to predict the site or future development of acute coronary events, new invasive and non-invasive techniques are available to detect the different characteristics of coronary lesions that may be of some importance in predicting future events. Angiography, the traditional gold standard, can only detect advanced lesions and provide a measure of the degree of stenosis, which has little prognostic value. Other techniques, like intravascular ultrasound and angioscopy, can identify the presence of thrombotic lesions or even lipid rich, vulnerable plaques. However, their ability to predict subsequent acute events has not been fully evaluated. Ultrafast computed tomography is useful in measuring the calcium content of coronary arteries. This non-invasive test may be useful for predicting the presence of coronary artery disease but clinical studies have yet to confirm that there is a good correlation between calcium content and plaque vulnerability. Furthermore, on the basis of intravascular ultrasound data, unstable (acute) lesions are less likely to be calcified than stable coronary lesions. Magnetic resonance imaging techniques in the future may be able to image vulnerable plaques by characterising the various components of the plaque in terms of lipid, fibrous, and thrombotic material. More data on this technique will be available in the future. ${ }^{11}$

As acute syndromes are the end result of thrombus formation on a disrupted or eroded atherosclerotic plaque with impaired ability to dilate because of endothelial dysfunction, several potential treatments are available for interrupting these processes. Traditional antianginal treatment will not significantly alter these factors. In addition, angioplasty or bypass surgery has not been shown to alter the future occurrence of acute myocardial infarction. Nevertheless, the use of medical treatments like aspirin and other potent antiplatelet agents to decrease thrombus formation, cholesterol lowering strategies in appropriate patients to stabilise plaques and improve endothelial function, and oestrogen treatment in postmenopausal women are examples of how we might positively change the intracoronary milieu of the patient. More research in these areas is needed. Additionally, we have to be able to identify and treat that subset of patients with vulnerable plaques with or without known risk factors, and with or without symptoms, in whom the risk of future coronary events is unacceptably increased.

JOHN A AMBROSE

Mount SinaI Medical Center,

One Gustave L Levy Place,

New York, NY 10029-6574, USA

email:john_ambrose@smtplink.mssm.edu

1 Ambrose, JA. Coronary arteriographic analysis and angiographic morphology. $\mathcal{~ A m ~ C o l l ~ C a r d i o l ~ 1 9 8 9 ; 1 3 : 1 4 9 2 - 4 . ~}$

2 Ambrose JA, Tannenbaum MA, Alexopoulos D, et al. Angiographic progression of coronary artery disease and the development of myocardial infarction. $7 \mathrm{Am}$ Coll Cardiol 1988;12:56-62.

3 Little WC, Constantinescu MS, Applegate RJ, et al. Can coronary angiography predict the site of a subsequent myocardial infarction in patients with phy predict the site of a subsequent myocardial infarction in patients with
mild to moderate coronary artery disease? Circulation $1988 ; 78: 1157-66$.

4 Mulcahy D, Husain S, Zalos G, et al. Ischemia during ambulatory monitoring as a prognostic indicator in patients with stable coronary artery disease. ҒAMA 1997;277:318-24.

5 Kannel WB, Manning F. Natural history of angina pectoris in the Framingham study. Prognosis and survival. Am f Cardiol 1972;29:154-63.

6 Falk E, Shah PK, Fuster V. Coronary plaque disruption. Circulation 1995;92:657-71

7 Libby P. Molecular basis of the acute coronary syndromes. Circulation 1995; 91:2844-50

8 Brown BG, Gallery CA, Badger RS, et al. Incomplete lysis of thrombus in the moderate underlying atherosclerotic lesion during intracoronary infusion of streptokinase for acute myocardial infarction: quantitative angiinfusion of streptokinase for acute myocardial infarction
ographic observations. Circulation 1986;73:653-61.

9 Mann JM, Davies MJ. Vulnerable plaque: relation of characteristics to degree of stenosis in human coronary arteries. Circulation 1996;94:928-31.

10 Nakagomi A, Celermajer DS, Lumley T, et al. Angiographic severity of coronary narrowing is a surrogate marker for the extent of coronary atherosclerosis. Am f Cardiol 1996;78:516-19.

11 Toussaint JF, LaMuraglia GM, Southern JF, et al. Magnetic resonance images lipid, fibrous, calcified, hemorrhagic, and thrombotic components of human atherosclerosis in vivo. Circulation 1996;94:932-8. 